\title{
Free-space Optical Communications Using On-off Keying and Source Information Transformation
}

\author{
Luanxia Yang, Bingcheng Zhu, Julian Cheng, Senior Member, IEEE, Jonathan F. Holzman, Member, IEEE
}

\begin{abstract}
Free-space optical communication using on-off keying (OOK) and source information transformation is proposed. It is shown that source information transformation allows the proposed system to detect the OOK signal without requiring the knowledge of instantaneous channel state information and the probability density function (pdf) of the turbulence model. Analytical expressions are derived for the pdf of the detection threshold, and an upper bound is obtained on the average bit error rate (BER). Numerical studies show that the proposed system can achieve comparable performance to the idealized adaptive detection system, with a greatly reduced level of implementation complexity and a signal-to-noise ratio performance loss of only $1.8 \mathrm{~dB}$ at a BER of $1 \times 10^{-9}$ for a lognormal turbulence channel with $\sigma=0.25$.
\end{abstract}

\section{INTRODUCTION}

Free-space optical (FSO) communication systems have important advantages over their radio frequency counterparts. For example, FSO systems can offer broadband operation, high link security, and freedom from spectral license regulations. Optical signals that are transmitted over free-space are subject to amplitude and phase distortion. Due to transient inhomogeneities of atmospheric temperature and pressure [1], [2], however, and the resulting scintillation or fading is a major cause of performance degradation for FSO systems. The performance degradation is especially pronounced for FSO systems using irradiance modulation and direct detection (IM/DD) with on-off keying (OOK) and fixed detection thresholds, which are non-adaptive and unoptimized [3], [4]. Such systems can produce irreducible error floors if operation is extended to high signal-to-noise ratios (SNRs) [5].

Attempts to overcome the irreducible error floors of OOK IM/DD systems have focused on the application of adaptive detection thresholds. The standard approach proposed for adaptive detection applies perfect knowledge of the instantaneous channel state information (CSI), as it uses the instantaneous SNR to detect each data symbol [2], [6]. This approach has obvious practical concerns for OOK IM/DD operation with nanosecond data symbol durations (i.e., Gbps rates) and millisecond turbulence coherence times, as rapid detection threshold adjustments are needed on the timescale of the millisecond turbulence coherence times [2], [7]-[9]. To accommodate these practical concerns, the electrical-SNR-optimized

Luanxia Yang, Julian Cheng and Jonathan F. Holzman are with School of Engineering, The University of British Columbia, Kelowna, BC, Canada V1V 1 V7 (e-mail: yangluanxia@hotmail.com, \{julian.cheng, jonathan.holzman\}@ubc.ca).

Bingcheng Zhu is with School of Information Science and Engineering, Southeast University, Nanjing, Jiangsu, China 210096 (e-mail: 230109122@seu.edu.cn) detection system was proposed in [10], [11]. Electrical-SNRoptimized detection thresholds only need to change over the especially long timescales, of seconds or minutes, over which a stationary turbulence channel assumption applies [12]. However, this method requires knowledge of the probability density function (pdf) of the turbulence channel, which increases the computation complexity.

In [13], the authors introduced pilot-symbol (PS) assisted modulation (PSAM) to mitigate the turbulence fading and improve the system performance. The PS provided the receiver with explicit turbulence fading references for detection, and this can be used to mitigate the effects of fading. However, PSAM can cause delays in the receiver as it is necessary to store the whole frame before decoding[14, Chap. 5]. In [15], it was demonstrated that an OOK IM/DD system could use two laser wavelengths at the transmitter and two photodetectors at the receiver to work in a differential mode, and achieve excellent bit error rate (BER) performance with a detection threshold fixed at zero. Unfortunately, this scheme suffers from low throughput, as two lasers are used to transmit the same information in each symbol duration.

In this work, we propose an alternative scheme that uses two (or more) laser transmitters. Such a scheme can improve the throughput beyond that of [15]. Moreover, the proposed scheme does not require knowledge of the instantaneous CSI and pdf of the turbulence channel, as the receivers have explicit turbulence fading references for detection. It is shown that such a system provides good BER performance without irreducible error floors.

The remainder of this work is organized as follows. Section II describes the system and channel models. Section III derives the pdf of the detection threshold. Section IV obtains an upper bound on the average BER. Numerical results and discussions are presented in Section V. Finally, Section VI makes some concluding remarks.

\section{SyStem AND CHANNEL MODELS}

We consider an IM/DD system with $M$ laser source transmitters and $M$ photodetectors operating through atmospheric turbulence channels. The operation of the proposed scheme is as follows. At the transmitter, which is shown in Fig. 1, there are $M$ distinct optical wavelengths, $\lambda_{1}, \lambda_{2}, \ldots, \lambda_{M}$, assigned to the $M$ laser transmitters. Each wavelength is used to transmit an independent information sequence, with source information transformation used to ensure that one or more lasers transmit bit " 1 " during each symbol duration. When $M=2$, for example, the proposed system can almost double 


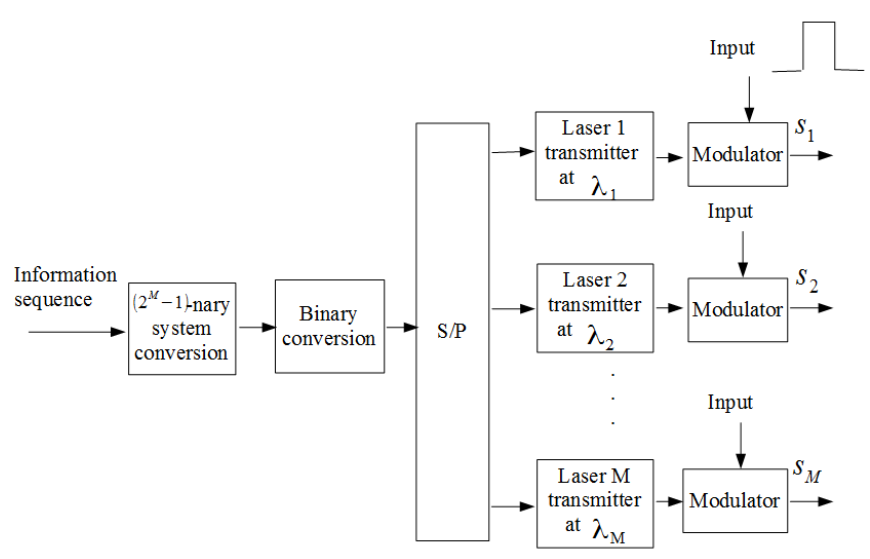

Fig. 1. Block diagram of the transmitter for the system using source information transformation.

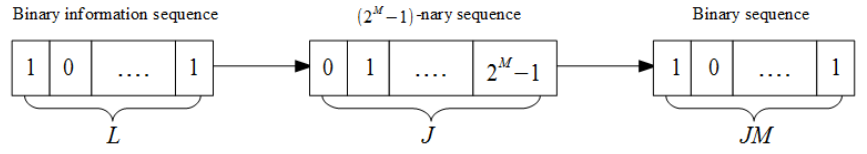

Fig. 2. Block diagram of the source information transformation. A binary information sequence of length $L$ is converted to a $\left(2^{M}-1\right)$-nary information sequence of length $J$, and this $\left(2^{M}-1\right)$-nary information sequence of length $J$ is mapped to a binary information sequence of length $J M$.

the multiplexing gain achieved in the system of [15] using double-laser differential signaling.

For source information transformation, we first convert a binary information sequence of length $L$ to a $\left(2^{M}-1\right)$-nary information sequence of length $J$ as shown in Fig. 2. This mapping can be written as

$$
T_{1}:\{0,1\}^{L} \rightarrow\left\{0, \ldots, 2^{M}-1\right\}^{J} .
$$

Then we map each element of the $\left(2^{M}-1\right)$-nary sequence into an $M$-bit binary sequence that does not contain the allzero binary sequence. The resulting $M$-bit binary sequence after the serial-to-parallel conversion determines, among the $M$ transmitted lasers, which link transmits bit " 0 " and which link transmits bit "1". For example, when $M=3$, we map the seven elements of the 7 -nary sequence $(0,1,2,3,4,5,6)$ to the binary sequence $\{001,010,011,100,101,110,111\}$. (Note here that the all-zero binary sequence is avoided.) This mapping can be written as

$$
T_{2}:\left\{0, \ldots, 2^{M}-1\right\}^{J} \rightarrow\{0,1\}^{J M} .
$$

The mapping described in (1) and (2), which we call source information transformation, will ensure that the $M$ received signals have an explicit turbulence fading reference for detection in each symbol duration, meaning that at least one laser is on (ie., at least one bit "1" is transmitted). Each bit "1" suffers turbulence distortion and therefore be used as a turbulence fading reference.

It is desirable to select values of $L$ and $J$ that make the mapping of $T_{1}$ be a one-to-one mapping, i.e.,

$$
2^{L}=\left(2^{M}-1\right)^{J} .
$$

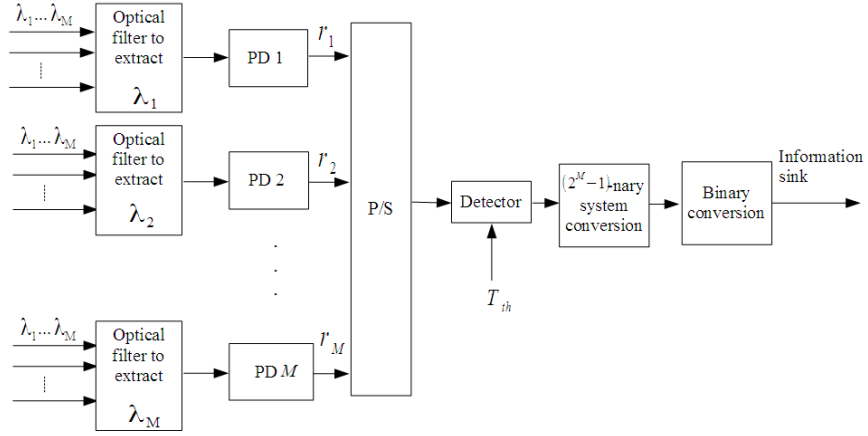

Fig. 3. Block diagram of the receiver for the system using source information transformation.

However, the above equality is difficult to achieve in practice for arbitrary values of $L$ and $J$. Thus, to approximate the ideal case of (3), we consider

$$
\begin{aligned}
& \min _{(L, J)}\left[\left(2^{M}-1\right)^{J}-2^{L}\right] \\
& \text { Subject to } 2^{L} \leq\left(2^{M}-1\right)^{J} .
\end{aligned}
$$

Since there might be more than one pair $(L, J)$ that satisfies (4), we will choose the smallest pair $(L, J)$ for our system, i.e., the value of $L+J$ that is the smallest among the pairs satisfying (4). This is done to minimize the system delay. For example, when $M=3$, we select $L=14$ and $J=5$ by using a computer search.

At the receiver, as shown in Fig. 3, diffractive optical elements and/or narrowband optical filters are used to separate the wavelengths for detection of the $M$ transmitted signals. In Fig. 3, we use the acronym PD to represent the photodetector. After the $M$ parallel photodetectors and the parallel-to-serial conversion, in each symbol duration, a value of one-half of the largest received signal is used to define the detection threshold for the $M$ received signals. If all of the $M$-bit binary sequence are demodulated as bit " 0 ", which may happen due to the noise, this is an incorrect decision (since an all-zero binary sequence is not transmitted for our system), and we will assume the source transmits $00 \ldots 01$. The demodulated $J M$-bit binary sequence will be mapped to a $\left(2^{M}-1\right)$-nary sequence of length $J$, and then this $\left(2^{M}-1\right)$-nary sequence of length $J$ will ultimately be converted back to a binary information sequence of length $L$.

At the $m$ th transmitter, the baseband signal to be transmitted can be expressed as

$$
s_{m}(t)=\sum_{i} a_{i, m} g_{m}\left(t-i T_{p}\right), \quad m=1,2, \ldots, M
$$

where $a_{i, m} \in\{0,1\}$ is the $i$ th data in the $m$ th transmitter, and $T_{p}$ is the symbol duration. In (5), pulse shaping in the $m$ th transmitter is defined as $g_{m}(t)=1$ for $0<t<T_{p}$, and $g_{m}(t)=0$ otherwise. These $M$ signals are transmitted through atmospheric turbulence channels and are distorted by a multiplicative process $I(u, t)$. We have assumed that the channel fading is the same for all the wavelengths in each symbol duration. This assumption can be achieved by ensuring that the transmitter wavelengths are sufficiently close to each 
other (being separated only by tens of nanometers). This ensures that the transmitter beams are spatially overlapped and experience the same atmospheric turbulence distortion [15].

At the $m$ th receiver, the received signal after the photodetection can be written as

$$
\begin{array}{r}
r_{m}(t)=R I(u, t) \sum_{i} a_{i, m} g_{m}\left(t-i T_{p}\right)+n_{m}(t), \\
m=1,2, \ldots, M .
\end{array}
$$

Without loss of generality, the photodetector responsivity $R$ is assumed to be unity. In $(6), I(u, t)$ is assumed to be a stationary random process for signal scintillation caused by atmospheric turbulence, and $n_{m}(t)$ is additive white Gaussian noise (AWGN) due to thermal noise and/or ambient shot noise in $m$ th receiver. Using a p-i-n photodiode and following [10], the shot noise is assumed to be dominated by ambient shot noise. (Both ambient shot noise and thermal noise are statistically independent of the desired signal.) The total noise power is $\sigma_{g}^{2}=\sigma_{s}^{2}+\sigma_{T}^{2}$, where $\sigma_{s}^{2}$ and $\sigma_{T}^{2}$ denote the respective variances of the ambient shot noise power and the thermal noise power.

The $m$ th received signal is sampled at time $T_{p}$. The sample $I\left(u, t=T_{p}\right)$ is a $\mathrm{RV} I$, and the sample $n_{m}\left(t=T_{p}\right)$ is an AWGN RV $n_{m}$ having zero mean and variance $\sigma_{g}^{2}$. When bit " 0 " is transmitted, $s_{0}$ becomes true and the laser is off. The demodulation sample is $\left.r_{m}\right|_{s_{0}}=n_{m}$. When bit " 1 " is transmitted, $s_{1}$ becomes true and the laser is on. The demodulation sample is $\left.r_{m}\right|_{s_{1}}=I+n_{m}$.

\section{The Probability Density Function of the DETECTION THRESHOLD}

With perfect knowledge of the instantaneous CSI, the minimum error probability is provided by the maximum-likelihoodbased decision threshold which can be expressed by [11]

$$
T_{t h}=\frac{\sigma_{0}\left(I_{0}+I_{1}\right)+\sigma_{1} I_{0}}{\sigma_{0}+\sigma_{1}}
$$

where $\sigma_{1}$ and $\sigma_{0}$ are the standard deviations of the noise currents for bits " 1 " and " 0 ", respectively, and $I_{1}$ and $I_{0}$ are averages of the generated currents at the receiver for bits " 1 " and " 0 ". For simplicity, we assume $\sigma_{0}=\sigma_{1}=\sigma_{g}, I_{0}=0$ and $I_{1}=I$. The maximum-likelihood-based detection threshold is $T_{t h}=I / 2$, which is an adaptive detection threshold, as it varies with the fading coefficient. Note that this approach is complex to realize in practice, as it requires perfect knowledge of the instantaneous CSI for each symbol detection. However, when the average SNR (denoted by $\gamma$ ) approaches infinity, or for a noiseless system, we have

$$
\lim _{\gamma \rightarrow \infty} \max \left\{r_{1}, r_{2}, \ldots, r_{M}\right\}=I .
$$

Thus, we can intuitively set the detection threshold for the system to be

$$
T_{t h}=\frac{\max \left\{r_{1}, r_{2}, \ldots, r_{M}\right\}}{2} .
$$

The most important feature of the detection threshold proposed in (9) is that it only depends on the received signal, and unlike an ideal optimized OOK detection scheme, an estimate of the CSI is not required.

We now derive the pdf of the detection threshold $T_{t h}$ in (9). In a symbol duration, we first consider the case for which $k$ branches transmit bit " 1 ", where $k=1,2, \ldots, M$, and the rest of the $M-k$ branches transmit bit " 0 ". Without loss of generality, we assume the first $k$ branches transmit bit " 1 ", and the rest of the $M-k$ branches transmit bit " 0 ". The conditional pdf of $T_{t h}$ can be written as

$$
\begin{aligned}
& f_{T_{t h}}\left(t_{t h} \mid I, k\right) \\
& =2 k \Phi\left(\frac{2 t_{t h}-I}{\sigma_{g}}\right)^{k-1} \Phi\left(\frac{2 t_{t h}}{\sigma_{g}}\right)^{M-k} f_{N}\left(2 t_{t h}-I\right) \\
& +2(M-k) \Phi\left(\frac{2 t_{t h}-I}{\sigma_{g}}\right)^{k} \Phi\left(\frac{2 t_{t h}}{\sigma_{g}}\right)^{M-k-1} f_{N}\left(2 t_{t h}\right)
\end{aligned}
$$

where $\Phi(x)=\int_{-\infty}^{x} \frac{1}{\sqrt{2 \pi}} \exp \left(-\frac{r^{2}}{2}\right) d r$ is the cumulative distribution function of a standard Gaussian RV, and $f_{N}(x)=$ $\frac{1}{\sqrt{2 \pi} \sigma_{g}} \exp \left(-\frac{x^{2}}{2 \sigma_{g}^{2}}\right)$ denotes the noise pdf. The detailed derivations of (10) are given in the Appendix. The pdf of $T_{t h}$ conditioned on $I$ can be obtained as

$$
\begin{aligned}
& f_{T_{t h}}\left(t_{t h} \mid I\right)=\sum_{k=1}^{M} f_{T_{t h}}\left(t_{t h} \mid I, k\right) p(k) \\
& =\frac{f_{N}\left(2 t_{t h}-I\right)}{2^{M-1}}\left\{M\left[\Phi\left(\frac{2 t_{t h}}{\sigma_{g}}\right)+\Phi\left(\frac{2 t_{t h}-I}{\sigma_{g}}\right)\right]^{M-1}\right. \\
& \left.+\left(\Phi\left(\frac{2 t_{t h}}{\sigma_{g}}\right)\right)^{M-1}\right\}+\frac{f_{N}\left(2 t_{t h}\right)}{2^{M-1}} \\
& \times\left\{M\left(\Phi\left(\frac{2 t_{t h}}{\sigma_{g}}\right)+\Phi\left(\frac{2 t_{t h}-I}{\sigma_{g}}\right)\right)^{M-1}\right. \\
& \left.+\left(\Phi\left(\frac{2 t_{t h}}{\sigma_{g}}\right)\right)^{M-2}\left[(M-1) \Phi\left(\frac{2 t_{t h}-I}{\sigma_{g}}\right)-M \Phi\left(\frac{2 t_{t h}}{\sigma_{g}}\right)\right]\right\}
\end{aligned}
$$

where $p(k)=\left(\begin{array}{c}M \\ k\end{array}\right) / 2^{M}$ is the probability that there are $k$ branches transmitting bit " 1 " values. Averaging (11) with respect to the fading coefficient $I$, one can obtain the pdf of $T_{t h}$ as

$$
f_{T_{t h}}\left(t_{t h}\right)=E_{I}\left[f_{T_{t h}}\left(t_{t h} \mid I\right)\right]
$$

where $E_{I}[\cdot]$ represents the statistical expectation with respect to $I$.

\section{The Upper Bound on the Average BER}

As it is challenging to find the exact BER expression for our proposed system, we will find an upper bound on the average BER. For expository purposes, we first analyze the error caused in the detection process. The error in the detection process, before the $\left(2^{M}-1\right)$-nary sequence conversion at the receiver, is the turbulence induced error when the binary sequence is transmitted through the turbulence channel. We then analyze the average BER of the output binary sequence. 


\section{A. Error in the Detection Process at the Receiver}

Without loss of generality, it is assumed that the first $k$ branches transmit bit " 1 ", and the rest of the $M-k$ branches transmit bit " 0 ". The detection threshold becomes

$$
\tilde{T}_{t h}=\frac{\max \left\{I+n_{1}, \ldots, I+n_{k}, n_{k+1}, \ldots, n_{M}\right\}}{2} .
$$

We define $\mathbf{N}=[n[1] \quad \ldots \quad n[M]]^{T}$ as the noise vector, and $\mathbf{N}_{k} \odot=\left[\begin{array}{llllll}n[1] & \ldots & n[k-1] & n[k+1] & \ldots & n[M]\end{array}\right]^{T}$ as the noise vector without the $k$ th noise component $n_{k}$. The probability of having incorrect detection in one or more links can be written as

$$
\begin{aligned}
& P\left(e_{2} \mid k\right) \\
& =\frac{1}{M}\left\{E_{\mathbf{N}_{1} \odot}\left[E_{I}\left[P\left(I+n_{1}<\tilde{T}_{t h} \mid \mathbf{N}_{1 \odot}, I\right)\right]\right]\right. \\
& +\ldots+E_{\mathbf{N}_{k} \odot}\left[E_{I}\left[P\left(I+n_{k}<\tilde{T}_{t h} \mid \mathbf{N}_{k} \odot, I\right)\right]\right] \\
& +E_{\mathbf{N}_{k+1} \odot}\left[E_{I}\left[P\left(n_{k+1}>\tilde{T}_{t h} \mid \mathbf{N}_{k+1} \odot, I\right)\right]\right] \\
& \left.+\ldots+E_{\mathbf{N}_{M} \odot}\left[E_{I}\left[P\left(n_{M}>\tilde{T}_{t h} \mid \mathbf{N}_{M} \odot, I\right)\right]\right]\right\} .
\end{aligned}
$$

Since all components of the noise vector $\mathbf{N}$ are independent and identically distributed (i.i.d.), for $k_{1} \neq k_{2}$, where $k_{1}, k_{2} \in$ $\{1,2, \ldots, M\}$, we have

$$
\begin{aligned}
& E_{\mathbf{N}_{k_{1}} \odot}\left[E _ { I } \left[P \left(I+n_{k_{1}}<\tilde{T}_{t h} \mid \mathbf{N}_{\left.\left.\left.k_{1} \odot, I\right)\right]\right]}\right.\right.\right. \\
= & E_{\mathbf{N}_{k_{2}} \odot}\left[E _ { I } \left[P \left(I+n_{k_{2}}<\tilde{T}_{t h} \mid \mathbf{N}_{\left.\left.\left.k_{2} \odot, I\right)\right]\right]}\right.\right.\right.
\end{aligned}
$$

and

$$
\begin{aligned}
& E_{\mathbf{N}_{k_{1}} \odot}\left[E _ { I } \left[P \left(n_{k_{1}}>\tilde{T}_{t h} \mid \mathbf{N}_{\left.\left.\left.k_{1} \odot, I\right)\right]\right]}\right.\right.\right. \\
= & E_{\mathbf{N}_{k_{2}} \odot}\left[E _ { I } \left[P \left(n_{k_{2}}>\tilde{T}_{t h} \mid \mathbf{N}_{\left.\left.\left.k_{2} \odot, I\right)\right]\right] .}\right.\right.\right.
\end{aligned}
$$

Thus, the probability of having incorrect detection in one or more links can be written as

$$
\begin{aligned}
& P\left(e_{2} \mid k\right)=\frac{k}{M} E_{\mathbf{N}_{1} \odot}\left[E_{I}\left[P\left(I+n_{1}<\tilde{T}_{t h} \mid \mathbf{N}_{1 \odot}, I\right)\right]\right] \\
& +\frac{M-k}{M} E_{\mathbf{N}_{M} \odot}\left[E_{I}\left[P\left(n_{M}>\tilde{T}_{t h} \mid \mathbf{N}_{M \odot}, I\right)\right]\right] .
\end{aligned}
$$

The first term in (17) can be upper-bounded as (18) on the top of the next page. The second term in (17) can be upperbounded as (19) on the next page. Subtituting (18) and (19) into (17), we have

$$
\begin{aligned}
& P\left(e_{2} \mid k\right)<\frac{k}{M}\left\{E_{I}\left[P\left(n_{1}<-I\right)\right]+(k-1)\right. \\
& \times E_{n_{2}}\left[E_{I}\left[P\left(n_{1}<\frac{n_{2}-I}{2} \mid n_{2}, I\right)\right]\right] \\
& \left.+(M-k) E_{n_{M}}\left[E_{I}\left[P\left(n_{1}<\frac{n_{M}}{2}-I \mid n_{M}, I\right)\right]\right]\right\} \\
& +\frac{M-k}{M} E_{n_{1}}\left[E_{I}\left[P\left(n_{M}>\frac{I+n_{1}}{2} \mid n_{1}, I\right)\right]\right] .
\end{aligned}
$$

The upper bound on the average BER for the binary sequence transmitted through the turbulence channel with $M$ transmit lasers is obtained as (21) on the next page. It is difficult to find a closed-form expression of (21), as it contains a double integral, however, this integral can be evaluated numerically with high accuracy.

\section{B. Average BER of the System}

At the transmitter, a binary sequence $a_{L} \ldots a_{2} a_{1}$ is converted into a $\left(2^{M}-1\right)$-nary sequence $h_{J} h_{J-1} \ldots h_{2} h_{1}$. This $\left(2^{M}-1\right)$-nary sequence of length $J$ is mapped to a binary sequence of length $J M$. At the receiver, after the demodulation, we will map a binary sequence of length $J M$ to a $\left(2^{M}-1\right)$ nary sequence $\hat{h}_{J} \hat{h}_{J-1} \ldots \hat{h}_{2} \hat{h}_{1}$. This $\left(2^{M}-1\right)$-nary sequence $\hat{h}_{J} \hat{h}_{J-1} \ldots \hat{h}_{2} \hat{h}_{1}$ is converted to a binary sequence, denoted as $\hat{a}_{L+1} \hat{a}_{L} \ldots \hat{a}_{2} \hat{a}_{1}$. We comment that when a $\left(2^{M}-1\right)$-nary sequence of length $J$ will be converted to a binary sequence of length $L+1$; however, at the transmitter, we convert a binary information sequence of length $L$ to a $\left(2^{M}-1\right)$-nary information sequence of length $J$. Thus, at the receiver, we will ignore $\hat{a}_{L+1}$ and use $\hat{a}_{L} \ldots \hat{a}_{2} \hat{a}_{1}$ as our binary information sequence.

To calculate the probability of having an incorrect decision in the binary sequence $\hat{a}_{L} \ldots \hat{a}_{2} \hat{a}_{1}$, for expository purposes, we consider $M=3$. When $M=3$, we will convert $L=14$ binary information bits $a_{14} \ldots a_{2} a_{1}$ to a 7-nary sequence with a length of $J=5\left(h_{5} h_{4} h_{3} h_{2} h_{1}\right)$. Then we map the 7-nary sequence of length of $J=5$ into a binary sequence of length $J M=15$. At the receiver, after the demodulation, we map each sequence of $M=3$ binary bits to an element of the 7-nary sequence. The mapping can be seen as follows:

$$
\begin{aligned}
& 000 \rightarrow 0,001 \rightarrow 0,010 \rightarrow 1,011 \rightarrow 2, \\
& 100 \rightarrow 3,101 \rightarrow 4,110 \rightarrow 5,111 \rightarrow 6 .
\end{aligned}
$$

Tabel I shows the conditional probability of the received $\left(2^{M}-\right.$ $1)$-nary number $\hat{h}_{l}$ given the transmitted $\left(2^{M}-1\right)$-nary number $h_{l}$. The conditional probability in Tabel I can be calculated by using the bit error probability for the binary bit transmitted through the turbulence channel, $P_{e_{2}}$. For example, $P(0 \mid 0)=$ $P(000 \mid 001)+P(001 \mid 001)=\left(1-P_{e_{2}}\right)^{2} P_{e_{2}}+\left(1-P_{e_{2}}\right)^{3}$. Tabel II shows the conditional probability of the received $\left(2^{M}-1\right)$ nary number $\hat{h}_{l}$ given the transmitted $\left(2^{M}-1\right)$-nary number $h_{l}$, written in terms of $P_{e_{2}}$.

Since all elements of $\hat{h}_{5} \ldots \hat{h}_{2} \hat{h}_{1}$ and $h_{5} \ldots h_{2} h_{1}$ are independent, the conditional probability for the received $\left(2^{M}-1\right)$ nary sequence $\hat{h}_{5} \ldots \hat{h}_{2} \hat{h}_{1}$ given the transmitted $\left(2^{M}-1\right)$-nary sequence $h_{5} \ldots h_{2} h_{1}$ is

$$
\begin{aligned}
& P\left(\hat{h}_{5} \ldots \hat{h}_{2} \hat{h}_{1} \mid h_{5} \ldots h_{2} h_{1}\right) \\
= & \prod_{j=1}^{5} P\left(\hat{h}_{j} \mid h_{5} \ldots h_{2} h_{1}\right)=\prod_{j=1}^{5} P\left(\hat{h}_{j} \mid h_{j}\right) .
\end{aligned}
$$

The conditional probability of the received binary sequence $\hat{a}_{L} \ldots \hat{a}_{2} \hat{a}_{1}$ given the transmitted binary sequence $a_{L} \ldots a_{2} a_{1}$ can be written as

$$
\begin{aligned}
& P\left(\hat{a}_{14} \ldots \hat{a}_{2} \hat{a}_{1} \mid a_{L} \ldots a_{2} a_{1}\right) \\
& =P\left(\hat{h}_{5} \ldots \hat{h}_{2} \hat{h}_{1} \mid h_{5} \ldots h_{2} h_{1}\right)=\prod_{j=1}^{5} P\left(\hat{h}_{j} \mid h_{j}\right) .
\end{aligned}
$$

If the transmitted binary sequence is $a_{14} \ldots a_{2} a_{1}$, and the received binary sequence is $\hat{a}_{14} \ldots \hat{a}_{2} \hat{a}_{1}$, the conditional error probability of this system given $a_{14} \ldots a_{2} a_{1}$ and $\hat{a}_{14} \ldots \hat{a}_{2} \hat{a}_{1}$ is

$$
P\left(e \mid a_{14} \ldots a_{2} a_{1}, \hat{a}_{14} \ldots \hat{a}_{2} \hat{a}_{1}\right)=\frac{\sum_{l=1}^{14} a_{l} \oplus \hat{a}_{l}}{14}
$$




$$
\begin{aligned}
& P\left(I+n_{1}<\tilde{T}_{t h} \mid \mathbf{N}_{1 \odot}, I\right)=P\left(I+n_{1}<\frac{\max \left\{I+n_{1}, \ldots, I+n_{k}, n_{k+1}, \ldots, n_{M}\right\}}{2} \mid \mathbf{N}_{1 \odot}, I\right) \\
& =P\left(\left\{I+n_{1}<\frac{I+n_{1}}{2}\right\} \cup \ldots \cup\left\{I+n_{1}<\frac{I+n_{k}}{2}\right\}\right. \\
& \left.\cup\left\{I+n_{1}<\frac{n_{k+1}}{2}\right\} \cup \ldots \cup\left\{I+n_{1}<\frac{n_{M}}{2}\right\} \mid \mathbf{N}_{1} \odot, I\right) \\
& \leq P\left(I+n_{1}<\frac{I+n_{1}}{2} \mid I\right)+P\left(I+n_{1}<\frac{I+n_{2}}{2} \mid n_{2}, I\right)+\ldots \\
& +P\left(I+n_{1}<\frac{I+n_{k}}{2} \mid n_{k}, I\right)+P\left(I+n_{1}<\frac{n_{k+1}}{2} \mid n_{k+1}, I\right) \\
& +\ldots+P\left(I+n_{1}<\frac{n_{M}}{2} \mid n_{M}, I\right) \\
& =P\left(n_{1}<-I \mid I\right)+P\left(n_{1}<\frac{n_{2}-I}{2} \mid n_{2}, I\right)+\ldots+P\left(n_{1}<\frac{n_{k}-I}{2} \mid n_{k}, I\right) \\
& +P\left(n_{1}<\frac{n_{k+1}}{2}-I \mid n_{k+1}, I\right)+\ldots+P\left(n_{1}<\frac{n_{M}}{2}-I \mid n_{M}, I\right) \\
& =P\left(n_{1}<-I\right)+(k-1) P\left(n_{1}<\frac{n_{2}-I}{2} \mid n_{2}, I\right)+(M-k) P\left(n_{1}<\frac{n_{M}}{2}-I \mid n_{M}, I\right) \text {. } \\
& P\left(n_{M}>\tilde{T}_{t h} \mid \mathbf{N}_{M \odot}, I\right)=P\left(n_{M}>\frac{\max \left\{I+n_{1}, \ldots, I+n_{k}, n_{k+1}, \ldots, n_{M}\right\}}{2} \mid \mathbf{N}_{M \odot}, I\right) \\
& =P\left(\left\{n_{M}>\frac{I+n_{1}}{2}\right\} \cap \ldots \cap\left\{n_{M}>\frac{I+n_{k}}{2}\right\}\right. \\
& \left.\cap\left\{n_{M}>\frac{n_{k+1}}{2}\right\} \cap \ldots \cap\left\{n_{M}>\frac{n_{M}}{2}\right\} \mid \mathbf{N}_{M \odot}, I\right) \\
& \leq P\left(n_{M}>\frac{I+n_{1}}{2} \mid n_{M}, I\right) \text {. }
\end{aligned}
$$

$$
\begin{aligned}
& P_{e_{2}}=\sum_{k=1}^{M} P\left(e_{2} \mid k\right) p(k) \\
< & \sum_{k=1}^{M} \frac{\left(\begin{array}{c}
M \\
k
\end{array}\right)}{2^{M}-1}\left\{\frac { k } { M } \left[E_{I}\left[P\left(n_{1}<-I\right)\right]+(k-1) E_{n_{2}}\left[E_{I}\left[P\left(n_{1}<\frac{n_{2}-I}{2} \mid n_{2}, I\right)\right]\right]\right.\right. \\
& \left.+(M-k) E_{n_{M}}\left[E_{I}\left[P\left(n_{1}<\frac{n_{M}}{2}-I \mid n_{M}, I\right)\right]\right]+\frac{M-k}{M} E_{n_{1}}\left[E_{I}\left[P\left(n_{M}>\frac{I+n_{1}}{2} \mid n_{1}, I\right)\right]\right]\right\} \\
= & \sum_{k=1}^{M} \frac{\left(\begin{array}{c}
M \\
k
\end{array}\right)}{2^{M}-1}\left\{\frac { k } { M } \left(E_{I}\left[Q\left(\frac{I}{\sigma_{g}}\right)\right]+(k-1) E_{n_{2}}\left[E_{I}\left[Q\left(\frac{I-n_{2}}{2 \sigma_{g}}\right)\right]\right]\right.\right. \\
& \left.\left.+(M-k) E_{n_{M}}\left[E_{I}\left[Q\left(\frac{2 I-n_{M}}{2 \sigma_{g}}\right)\right]\right]\right)+\frac{M-k}{M} E_{n_{1}}\left[E_{I}\left[Q\left(\frac{I+n_{1}}{2 \sigma_{g}}\right)\right]\right]\right\} .
\end{aligned}
$$

TABLE I

CONDITIONAL PROBABILITY OF THE RECEIVED $\left(2^{M}-1\right)$-NARY NUMBER $\hat{h}_{l}$ GIVEN THE TRANSMitTED $\left(2^{M}-1\right)$-NARY NUMBER $h_{l}$.

\begin{tabular}{|c|c|c|c|c|c|c|c|}
\hline \hline$\hat{h}_{l}$ & 0 & 1 & 2 & 3 & 4 & 5 & 6 \\
\hline$P\left(\hat{h}_{l} \mid h_{l}=0\right)$ & $P(0 \mid 0)$ & $P(1 \mid 0)$ & $P(2 \mid 0)$ & $P(3 \mid 0)$ & $P(4 \mid 0)$ & $P(5 \mid 0)$ & $P(6 \mid 0)$ \\
\hline$P\left(\hat{h}_{l} \mid h_{l}=1\right)$ & $P(0 \mid 1)$ & $P(1 \mid 1)$ & $P(2 \mid 1)$ & $P(3 \mid 1)$ & $P(4 \mid 1)$ & $P(5 \mid 1)$ & $P(6 \mid 1)$ \\
\hline$P\left(\hat{h}_{l} \mid h_{l}=2\right)$ & $P(0 \mid 2)$ & $P(1 \mid 2)$ & $P(2 \mid 2)$ & $P(3 \mid 2)$ & $P(4 \mid 2)$ & $P(5 \mid 2)$ & $P(6 \mid 2)$ \\
\hline$P\left(\hat{h}_{l} \mid h_{l}=3\right)$ & $P(0 \mid 3)$ & $P(1 \mid 3)$ & $P(2 \mid 3)$ & $P(3 \mid 3)$ & $P(4 \mid 3)$ & $P(5 \mid 3)$ & $P(6 \mid 3)$ \\
\hline$P\left(\hat{h}_{l} \mid h_{l}=4\right)$ & $P(0 \mid 4)$ & $P(1 \mid 4)$ & $P(2 \mid 4)$ & $P(3 \mid 4)$ & $P(4 \mid 4)$ & $P(5 \mid 4)$ & $P(6 \mid 4)$ \\
\hline$P\left(\hat{h}_{l} \mid h_{l}=5\right)$ & $P(0 \mid 5)$ & $P(1 \mid 5)$ & $P(2 \mid 5)$ & $P(3 \mid 5)$ & $P(4 \mid 5)$ & $P(5 \mid 5)$ & $P(6 \mid 5)$ \\
\hline$P\left(\hat{h}_{l} \mid h_{l}=6\right)$ & $P(0 \mid 6)$ & $P(1 \mid 6)$ & $P(2 \mid 6)$ & $P(3 \mid 6)$ & $P(4 \mid 6)$ & $P(5 \mid 6)$ & $P(6 \mid 6)$ \\
\hline \hline
\end{tabular}


TABLE II

THE CONDITIONAL PROBABILITy OF THE RECEIVED $\left(2^{M}-1\right)$-NARY NUMBER $\hat{h}_{l}$ GIVEN THE TRANSMitTed $\left(2^{M}-1\right)$-NARY NUMBER $h_{l}$.

\begin{tabular}{|c|c|c|c|c|c|c|c|}
\hline$\hat{h}_{l}$ & 0 & 1 & 2 & 3 & 4 & 5 & 6 \\
\hline$P\left(\hat{h}_{l} \mid h_{l}=0\right)$ & $\begin{array}{c}\left(1-P_{e_{2}}\right)^{2} P_{e_{2}} \\
+\left(1-P_{e_{2}}\right)^{3}\end{array}$ & $\left(1-P_{e_{2}}\right) P_{e_{2}}^{2}$ & $\left(1-P_{e_{2}}\right) P_{e_{2}}^{2}$ & $\left(1-P_{e_{2}}\right) P_{e_{2}}^{2}$ & $\left(1-P_{e_{2}}\right) P_{e_{2}}^{2}$ & $\left(1-P_{e_{2}}\right) P_{e_{2}}^{2}$ & $\left(1-P_{e_{2}}\right) P_{e_{2}}^{2}$ \\
\hline$P\left(\hat{h}_{l} \mid h_{l}=1\right)$ & $\begin{array}{l}\left(1-P_{e_{2}}\right)^{2} P_{e_{2}} \\
+\left(1-P_{e_{2}}\right) P_{e_{2}}^{2}\end{array}$ & $\left(1-P_{e_{2}}\right)^{3}$ & $\left(1-P_{e_{2}}\right) P_{e_{2}}^{2}$ & $\left(1-P_{e_{2}}\right) P_{e_{2}}^{2}$ & $P_{e_{2}}^{3}$ & $\left(1-P_{e_{2}}\right)^{2} P_{e_{2}}$ & $\left(1-P_{e_{2}}\right) P_{e_{2}}^{2}$ \\
\hline$P\left(\hat{h}_{l} \mid h_{l}=2\right)$ & $\begin{array}{l}\left(1-P_{e_{2}}\right)^{2} P_{e_{2}} \\
+\left(1-P_{e_{2}}\right) P_{e_{2}}^{2}\end{array}$ & $\left(1-P_{e_{2}}\right)^{2} P_{e_{2}}$ & $\left(1-P_{e_{2}}\right)^{3}$ & $P_{e_{2}}^{3}$ & $\left(1-P_{e_{2}}\right) P_{e_{2}}^{2}$ & $\left(1-P_{e_{2}}\right) P_{e_{2}}^{2}$ & $\left(1-P_{e_{2}}\right)^{2} P_{e_{2}}$ \\
\hline$P\left(\hat{h}_{l} \mid h_{l}=3\right)$ & $\begin{array}{l}\left(1-P_{e_{2}}\right)^{2} P_{e_{2}} \\
+\left(1-P_{e_{2}}\right) P_{e_{2}}^{2}\end{array}$ & $\left(1-P_{e_{2}}\right) P_{e_{2}}^{2}$ & $\overline{P_{e_{2}}^{3}}$ & $\left(1-P_{e_{2}}\right)^{3}$ & $\left(1-P_{e_{2}}\right)^{2} P_{e_{2}}$ & $\left(1-P_{e_{2}}\right)^{2} P_{e_{2}}$ & $\left(1-P_{e_{2}}\right) P_{e_{2}}^{2}$ \\
\hline$P\left(\hat{h}_{l} \mid h_{l}=4\right)$ & $\begin{array}{l}\left(1-P_{e_{2}}\right)^{2} P_{e_{2}} \\
+\left(1-P_{e_{2}}\right) P_{e_{2}}^{2} \\
\end{array}$ & $P_{e_{2}}^{3}$ & $\left(1-P_{e_{2}}\right) P_{e_{2}}^{2}$ & $\left(1-P_{e_{2}}\right)^{2} P_{e_{2}}$ & $\left(1-P_{e_{2}}\right)^{3}$ & $\left(1-P_{e_{2}}\right) P_{e_{2}}^{2}$ & $\left(1-P_{e_{2}}\right) P_{e_{2}}^{2}$ \\
\hline$P\left(\hat{h}_{l} \mid h_{l}=5\right)$ & $P_{e_{2}}^{3}+\left(1-P_{e_{2}}\right) P_{e_{2}}^{2}$ & $\left(1-P_{e_{2}}\right)^{2} P_{e_{2}}$ & $\left(1-P_{e_{2}}\right) P_{e_{2}}^{2}$ & $\left(1-P_{e_{2}}\right)^{2} P_{e_{2}}$ & $\left(1-P_{e_{2}}\right) P_{e_{2}}^{2}$ & $\left(1-P_{e_{2}}\right)^{3}$ & $\left(1-P_{e_{2}}\right) P_{e_{2}}^{2}$ \\
\hline$P\left(\hat{h}_{l} \mid h_{l}=6\right)$ & $P_{e_{2}}^{3}+\left(1-P_{e_{2}}\right) P_{e_{2}}^{2}$ & $\left(1-P_{e_{2}}\right) P_{e_{2}}^{2}$ & $\left(1-P_{e_{2}}\right)^{2} P_{e_{2}}$ & $\left(1-P_{e_{2}}\right) P_{e_{2}}^{2}$ & $\left(1-P_{e_{2}}\right) P_{e_{2}}^{2}$ & $\left(1-P_{e_{2}}\right) P_{e_{2}}^{2}$ & $\left(1-P_{e_{2}}\right)^{3}$ \\
\hline
\end{tabular}

where $\oplus$ implements an exclusive OR. Thus, the BER of this system with $M=3$ can be written as

$$
\begin{aligned}
& P(e)=\sum_{a_{14} \ldots a_{2} a_{1}, \hat{a}_{14} \ldots \hat{a}_{2} \hat{a}_{1}} P\left(e \mid a_{14} \ldots a_{2} a_{1}, \hat{a}_{14} \ldots \hat{a}_{2} \hat{a}_{1}\right) \\
& \times P\left(a_{14} \ldots a_{2} a_{1}, \hat{a}_{14} \ldots \hat{a}_{2} \hat{a}_{1}\right) \\
& =\sum_{a_{14} \ldots a_{2} a_{1}, \hat{a}_{14} \ldots \hat{a}_{2} \hat{a}_{1}} \frac{\sum_{l=1}^{14} a_{l} \oplus \hat{a}_{l}}{14} \\
& \times P\left(a_{14} \ldots a_{2} a_{1}, \hat{a}_{14} \ldots \hat{a}_{2} \hat{a}_{1}\right) \\
& =\sum_{a_{14} \ldots a_{2} a_{1}, \hat{a}_{14} \ldots \hat{a}_{2} \hat{a}_{1}} \frac{\sum_{l=1}^{14} a_{l} \oplus \hat{a}_{l}}{14} \\
& \times P\left(\hat{a}_{14} \ldots \hat{a}_{2} \hat{a}_{1} \mid a_{14} \ldots a_{2} a_{1}\right) P\left(a_{14} \ldots a_{2} a_{1}\right) .
\end{aligned}
$$

In (26), the elements of $a_{14} \ldots a_{2} a_{1}$ are independent, so we have $P\left(a_{14} \ldots a_{2} a_{1}\right)=\frac{1}{2^{14}}$. Substituting (24) into (26), we have

$$
\begin{aligned}
& P(e)=\sum_{a_{14} \ldots a_{2} a_{1}, \hat{a}_{14} \ldots \hat{a}_{2} \hat{a}_{1}} \frac{\sum_{l=1}^{14} a_{l} \oplus \hat{a}_{l}}{14} \\
& \times \prod_{j=1}^{5} P\left(\hat{h}_{j} \mid h_{j}\right) P\left(a_{14} \ldots a_{2} a_{1}\right) \\
& =\frac{1}{2^{14}} \sum_{a_{14} \ldots a_{2} a_{1}, \hat{a}_{14} \ldots \hat{a}_{2} \hat{a}_{1}} \frac{\sum_{l=1}^{14} a_{l} \oplus \hat{a}_{l}}{14} \prod_{j=1}^{5} P\left(\hat{h}_{j} \mid h_{j}\right) .
\end{aligned}
$$

In general, the BER for the proposed system with $M$ transmitted lasers can be written as

$$
P(e)=\frac{1}{2^{L}} \sum_{a_{L} \ldots a_{2} a_{1}, \hat{a}_{L} \ldots \hat{a}_{2} \hat{a}_{1}} \frac{\sum_{l=1}^{L} a_{l} \oplus \hat{a}_{l}}{L} \prod_{j=1}^{J} P\left(\hat{h}_{j} \mid h_{j}\right) .
$$

\section{Numerical Results}

In this section, the pdf of the detection threshold is first verified, and the BER performance of the proposed system is numerically studied.

In Fig. 4, the derived pdf of the detection threshold $T_{t h}$ is compared with the simulated pdf. For expository purposes, we

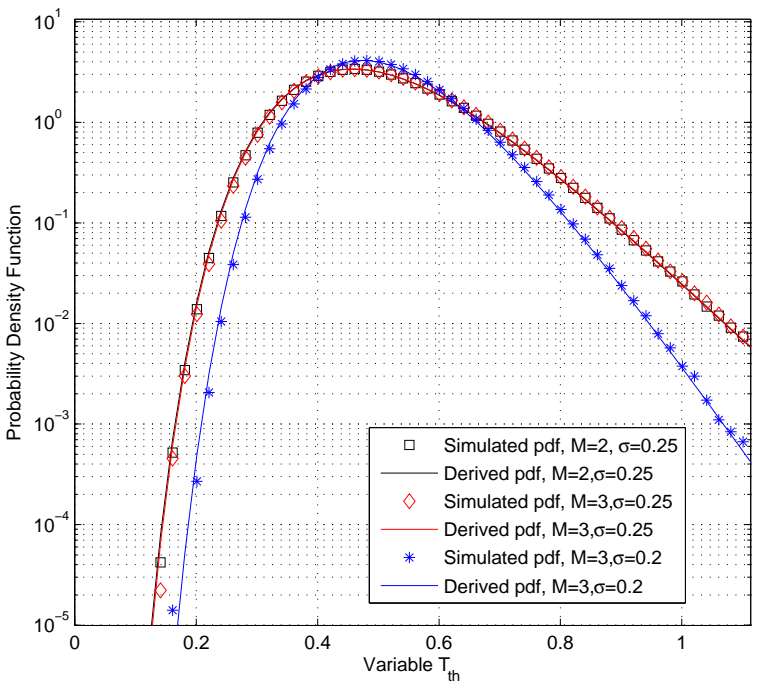

Fig. 4. Comparison of the derived and simulated pdfs for the detection threshold $T_{t h}$ over a lognormal fading channel with $(\sigma, M)=(0.25,3)$, $(0.2,3)$ and $(0.25,2)$.

let the parameters $(\sigma, M)=(0.25,3),(0.2,3)$ and $(0.25,2)$. The simulated pdf is obtained by using Monte Carlo computer simulations with $10^{4}$ trials. The derived pdf shows excellent agreement with the simulated pdf.

In Fig. 5, we plot the BER versus electrical SNR when the OOK IM/DD system uses a fixed detection threshold of $T_{t h}=0.5$. Note that an error floor appears in the large SNR regime. The system using source information transformation can eliminate the error floor, although its BER performance is worse than that of the OOK IM/DD system using fixed detection thresholds in low SNR regimes. This is due to the fact that a value of one-half of the largest received signal is used to define the detection threshold for the $M$ received signals in each symbol duration. This detection threshold is only optimum when the electrical SNR approaches infinity and/or there is no noise. In the low SNR regimes, the detection threshold is not an optimum detection threshold for our 


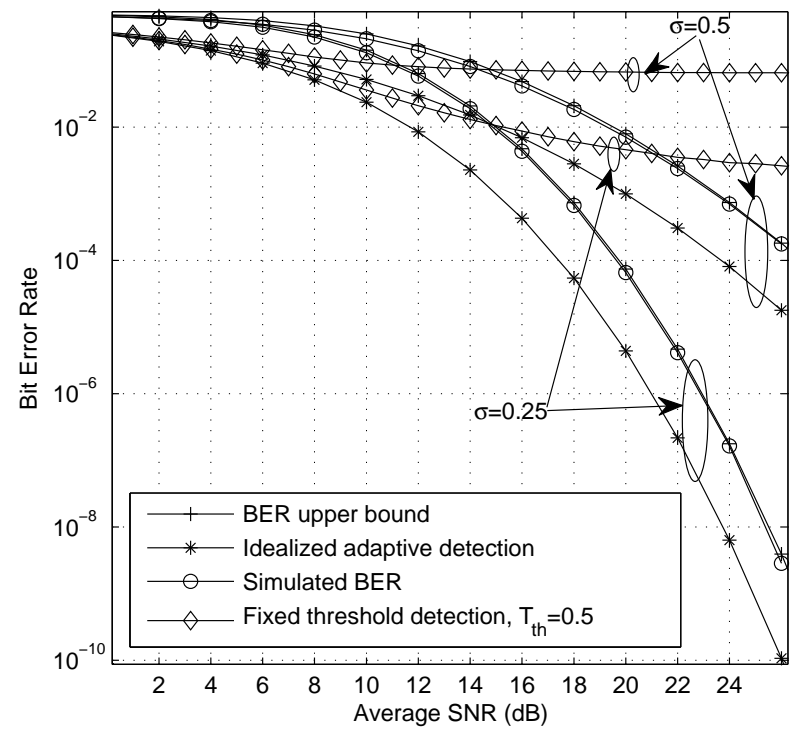

Fig. 5. The simulated BERs and BER upper bounds of the system using idealized adaptive detection thresholds, source information transformation, and fixed detection thresholds over lognormal turbulence channels (with $\sigma=0.25, \sigma=0.5$ and $M=3$ ).

proposed system, due to the noise influence, and the BER of our proposed system becomes worse than that of the OOK IM/DD system using a fixed detection threshold.

In Fig. 5, we also plot the upper bounds on the average BER for the proposed system over lognormal fading channels with different turbulence conditions. Simulated BER curves are also used to verify the analytical BER upper-bound solutions. The upper bound is tight when $M=3$. However, as we have used the union upper bound technique, it can be shown that the upper bound becomes loose with increasing $M$. It is seen from Fig. 5 that the OOK modulated system using idealized adaptive detection thresholds with a lognormal turbulence model having $\sigma=0.25$ requires an SNR of $24.8 \mathrm{~dB}$ to attain a BER of $1 \times 10^{-9}$, while the proposed system requires an SNR of $26.6 \mathrm{~dB}$ to achieve the same BER performance. Thus, the corresponding SNR penalty factor for the system using OOK and source information transformation in a lognormal turbulence channel with $\sigma=0.25$ is only $1.8 \mathrm{~dB}$ at BER of $1 \times 10^{-9}$. This performance difference can be factored into the ultimate FSO system design to offset the complexity of implementing systems with adaptive detection thresholds (and their need for knowledge of the instantaneous CSI).

\section{CONCLUSION}

FSO communication systems using OOK and source information transformation have been proposed. It was shown that such systems can achieve good BER performance without the need for knowledge of the instantaneous CSI and pdf of the turbulence model. We have derived an analytical expression for the pdf of the detection threshold and developed a tight upper bound on the average BER. Numerical studies ultimately showed that the system using coded wavelength multiplexing achieves comparable performance to the idealized adaptive detection system, with a greatly reduced level of implementation complexity (exhibiting an SNR penalty factor of only $1.8 \mathrm{~dB}$ at a BER of $1 \times 10^{-9}$, for a lognormal turbulence channel with $\sigma=0.25$ ).

\section{APPENDIX}

In a symbol duration, we first consider the case for which $k$ branches transmit bit " 1 ", where $k=1,2, \ldots, M$, and the rest of the $M-k$ branches transmit bit " 0 ". Without loss of generality, we assume the first $k$ branches transmit bit " 1 ", and the rest of the $M-k$ branches transmit bit " 0 ". The conditional cumulative distribution function of $T_{t h}$ can be written as (29) on the top of the next page. Since all the noise components $n_{1}, n_{2}, \ldots, n_{M}$ are assumed to be i.i.d., we have (30) on the top of the next page. It follows that

$$
\begin{aligned}
& F_{T_{t h}}\left(t_{t h} \mid I, k\right) \\
= & {\left[P\left(n_{1}<2 t_{t h}-I\right)\right]^{k}\left[P\left(n_{M}<2 t_{t h}\right)\right]^{M-k} } \\
= & {\left[\Phi\left(2 t_{t h}-I\right)\right]^{k}\left[\Phi\left(2 t_{t h}\right)\right]^{M-k} . }
\end{aligned}
$$

The pdf of $T_{t h}$ conditioned on $k$ branches transmitting bits " 1 "s and $I$ can be written as

$$
\begin{aligned}
& f_{T_{t h}}\left(t_{t h} \mid I, k\right)=\frac{d}{d t_{t h}} F_{T_{t h}}\left(t_{t h} \mid I, k\right) \\
& =2 k \Phi\left(\frac{2 t_{t h}-I}{\sigma_{g}}\right)^{k-1} \Phi\left(\frac{2 t_{t h}}{\sigma_{g}}\right)^{M-k} f_{N}\left(2 t_{t h}-I\right) \\
& +2(M-k) \Phi\left(\frac{2 t_{t h}-I}{\sigma_{g}}\right)^{k} \Phi\left(\frac{2 t_{t h}}{\sigma_{g}}\right)^{M-k-1} f_{N}\left(2 t_{t h}\right) .
\end{aligned}
$$

\section{REFERENCES}

[1] V. W. S. Chan, "Free-space optical communications," IEEE/OSA J. Lightwave Technol., vol. 24, pp. 4750-4762, Dec. 2006.

[2] J. H. Shapiro and R. C. Harney, "Burst-mode atmospheric optical communication," in Proc.1980 Nat. Telecommun. Conf., 1980, pp. 27.5.127.5.7.

[3] N. Chand, J. J. Loriz, A. J. Hunton, and B. M. Eteson, "Performance comparison of NRZ and RZ modulations with and without forward error corrections for free-space optical communication," in Proc. SPIE, vol. 5892, pp. 58920U-1-58920U-8, Sept. 2005.

[4] N. Liu, W. D. Zhong, Y. He, K. H. Heng, and T. H. Cheng, "Comparison of NRZ and RZ modulations in laser intersatellite communication systems," in Proc. 2008 Int. Conf. Advanced Infocomm Tech., Shenzhen, 2008, pp. 29-32.

[5] J. Li, J. Q. Liu, and D. P. Taylor, "Optical communication using subcarrier PSK intensity modulation through atmospheric turbulence channels," IEEE Trans. Commun., vol. 55, pp. 1598-1606, Aug. 2007.

[6] M. Tycz, M. W. Fitzmaurice, and D. A. Premo, "Optical communication system performance with tracking error induced signal fading," IEEE Trans. Commun., vol. 21, pp. 1069-1072, Sept. 1973.

[7] M. L. B. Riediger, R. Schober, and L. Lampe, "Fast multiple-symbol detection for free-space optical communications," IEEE Trans. Commun., vol. 57, pp. 1119-1128, Apr. 2009.

[8] M. L. B. Riediger, R. Schober, and L. Lampe, "Blind detection of onoff keying for free-space optical communications." in Proc. CCECE, Niagara Falls, Canada, 2008, pp. 1361-1364.

[9] H. R. Burris, et al., "Laboratory implementation of an adaptive thresholding system for free-space optical communication receivers with signal dependent noise," in Proc. SPIE, 2005, vol. 5892, pp. 1-20.

[10] X. Zhu and J. M. Kahn, "Free-space optical communication through atmospheric turbulence channels," IEEE Trans. Commun., vol. 50, pp. 1293-1300, Oct. 2002. 


$$
\begin{aligned}
F_{T_{t h}}\left(t_{t h} \mid I, k\right) & =P\left(\frac{\max \left\{I+n_{1}, \ldots, I+n_{k}, n_{k+1}, \ldots, n_{M}\right\}}{2}<t_{t h}\right) \\
& =P\left(\frac{I+n_{1}}{2}<t_{t h}, \ldots, \frac{I+n_{k}}{2}<t_{t h}, \frac{n_{k+1}}{2}<t_{t h}, \ldots, \frac{n_{M}}{2}<t_{t h}\right) \\
& =P\left(n_{1}<2 t_{t h}-I, \ldots, n_{k}<2 t_{t h}-I, n_{k+1}<2 t_{t h}, \ldots, n_{M}<2 t_{t h}\right) .
\end{aligned}
$$

$$
\begin{aligned}
& P\left(n_{1}<2 t_{t h}-I, \ldots, n_{k}<2 t_{t h}-I, n_{k+1}<2 t_{t h}, \ldots, n_{M}<2 t_{t h}\right) \\
& =P\left(n_{1}<2 t_{t h}-I\right) \ldots P\left(n_{k}<2 t_{t h}-I\right) P\left(n_{k+1}<2 t_{t h}\right) \ldots P\left(n_{M}<2 t_{t h}\right) \\
& =\left[P\left(n_{1}<2 t_{t h}-I\right)\right]^{k}\left[P\left(n_{M}<2 t_{t h}\right)\right]^{M-k} .
\end{aligned}
$$

[11] H. Moradi, H. H. Refai, and P. G. LoPresti, "Thresholding-based optimal detection of wireless optical signals," J. Opt. Comm. Net., vol. 2, pp. 689-700, Sept. 2010.

[12] D. A. DeWolf, "Are strong irradiance fluctuations log normal or Rayleigh distributed," J. Opt. Soc. Amer., vol. 57, pp. 787-797, Jun. 1967.

[13] X. Zhu and J. M. Kahn, "Pilot-symbol assisted modulation for correlated turbulent free-space optical channels." in Proc. SPIE, 2002, vol. 4489, pp. $138 \mathrm{C} 145$.

[14] J. G. Proakis, Digital Communications. New York: McGraw-Hill, 5th ed., 2008.

[15] M. Khalighi, F. Xu, Y. Jaafar, and S. Bourennane, "Double-laser differential signaling for reducing the effect of background radiation in free-space optical systems," IEEE/OSA J. Opt. Commun. Netw., vol. 3, pp. 145-154, Feb. 2011. 